\title{
Wire Haired Mini Dachshund
}

National Cancer Institute

\section{Source}

National Cancer Institute. Wire Haired Mini Dachshund. NCI Thesaurus. Code C53904.

The Wire Haired Mini Dachshund is a wire-haired variety of dachshund with a height up to 5 inches. 